\title{
A Time-Domain Nonlinear Simulation Method for Wave-Induced Motions of a Floating Body
}

\author{
by Masashi Kashiwagi*, Member Tetsushi Momoda** \\ Masaru Inada*
}

\begin{abstract}
Summary
A nonlinear calculation method based on the Mixed Eulerian Lagrangian method is presented for wave-induced motions of a 2-D floating body. Attention is placed on an effective calculation of the hydrodynamic force associated with the temporal derivative of the velocity potential in Bernoulli's pressure equation. Unlike other existing methods, the acceleration field can be computed simultaneously with the velocity field, which contributes greatly to reduction of the computation time. By use of Green's second identity, the new method is explained as an extension from the mode decomposition method, and close relations between the two methods are emphasized.

Computations are performed for a wall-sided model and a flared model, and numerical results of the waves at upwave and downwave positions and the body motions (sway, heave, and roll) are compared with corresponding experiments. The overall agreement is very good, confirming validity of the present method. Discussion is also made on the parametric oscillation in roll, observed for the flared model.
\end{abstract}

\section{Introduction}

Nonlinear calculation methods for the motion of a floating body in large-amplitude waves have been drawing attention in the seakeeping and ocean engineering research. Recently, great interest is placed on effects of the body geometry above the sea level, for which no information has been given by conventional linear theories. For this kind of research, the so-called Mixed Eulerian Lagrangian (MEL) method, initiated by Longuet-Higgins \& Cokelet $^{1)}$, is the most promising in the framework of the potential flow.

The MEL method has been studied by many researchers; recent references in this context are Cao et al. $^{2)}$, Tanizawa ${ }^{3), 4}$, Kashiwagi ${ }^{5)}, \mathrm{Wu} \&$ Eatock Taylor ${ }^{6)}$, and others cited therein. However, simulations of the wave-body interaction were not so successful, because of the difficulty in precise evaluation of the temporal derivative of the velocity potential, $\partial \phi / \partial t \equiv \phi_{t}$, appearing in Bernoulli's pressure equation.

The simplest way of evaluating $\phi_{t}$ is to use a backward finite-difference scheme in time. However, it is known that this scheme makes a solution inherently unstable, resulting in the breakdown of computations. An alternative is to solve the boundary-value problem

* Research Institute forApplied Mechanics, Kyushu University

** Oita Prefectural Government (Graduate School of Kyushu University during this research)

Received 2nd July 1998

Read at the Autumn meeting 12, 13th Nov. 1998 for $\phi_{t}$, which was initially proposed by Vinje \& Brevig ${ }^{7)}$. The difficulty in this case is that the body boundary condition for $\phi_{t}$ includes the acceleration of a body which is to be computed from the motion equation. The hydrodynamic force in the motion equation, in turn, requires the pressure integration and thus evaluation of $\phi_{t}$. To resolve this 'nested' situation, several ideas have been proposed. However, one common defect among those is that the boundary-value problem for $\phi_{t}$ must be solved after the velocity field is completely determined. That is, two boundary-value problems must be solved separately within one step of the time marching; this is a reason of large computation time in the wave-body interaction problem.

The present paper investigates a new indirect method, originally proposed by $\mathrm{Wu} \&$ Eatock Taylor ${ }^{6)}$, in which the boundary-value problem is solved for an artificial function $\psi_{i}$ (which is superficially similar to the radiation problem in the linear theory) instead of $\phi_{t}$ itself. In this method, the boundary-value problem for $\psi_{i}$ is the same in form but independent of the velocity field. Thus it can be solved simultaneously with the boundary-value problem for the velocity field, which contributes greatly to reduction of the computation time. Mathematical transformations in the new method are based on Green's second identity. Close relations are noted between the new method and the mode decomposition method known as a conventional method for the present problem.

Computations are performed for two different profiles of a 2-D floating body: wall-sided and flared models. Results of waves and the sway, heave, and roll motions 
of a floating body are compared with corresponding experiments. Discussion is made on validity and limitation of the present method and also on the parametric oscillation in roll observed for the flared model.

\section{Formulation for the Velocity Field}

As shown in Fig. 1, we consider a freely floating body on the free surface, subjected to a wave generated by a plunger-type wavemaker. The $x$-axis of the coordinate system coincides with the plane of the undisturbed free surface and the positive $y$-axis is taken downward. The bottom of water is finite and horizontal, with its depth denoted by $h$.

The gravitational center of a floating body is initially located at the point $\left(x_{G}, y_{G}\right)$, and subsequent displacement of that point due to wave-induced motions is denoted by $\xi_{1}(t), \xi_{2}(t)$, and $\alpha(t)$ for sway, heave, and roll, respectively. The body is moored by a weak spring to prevent from a large excursion in the horizontal direction.

The boundary of a fluid is denoted by $S_{0}$ on the $y$ axis, $S_{A}$ on a wavemaker, $S_{F}$ on the free surface, $S_{B}$ on a floating body, $S_{W}$ on a wave absorbing beach, and $S_{C}$ on a control surface which is placed artificially at some distance from a body to make the fluid domain bounded.

In the analyses that follow, all quantities are nondimensionalized in terms of the half beam of a floating body, $b$, the gravitational acceleration, $g$, and the fluid density, $\rho$.

Assuming the fluid to be inviscid with irrotational motion, the velocity potential $\phi$ can be introduced, satisfying the Laplace equation :

$\nabla^{2} \phi(x, y, t)=0$ in the fluid

The boundary conditions to be satisfied by the velocity potential are written as follows:

$$
\left.\begin{array}{l}
\frac{D x}{D t}=\frac{\partial \phi}{\partial x}, \frac{D y}{D t}=\frac{\partial \phi}{\partial y} \\
\frac{D \phi}{D t}=y+\frac{1}{2} \nabla \phi D \phi
\end{array}\right\} \quad \text { on } S_{F}
$$

$$
\begin{array}{ll}
\frac{\partial \phi}{\partial n}=\dot{y}_{0}(t) n_{2} & \text { on } S_{A} \\
\frac{\partial \phi}{\partial n}=\sum_{j=1}^{3} v_{j} n_{j} & \text { on } S_{B} \\
\frac{\partial \phi}{\partial n}=\frac{\partial \phi}{\partial x}=0 & \text { on } S_{0} \\
\frac{\partial \phi}{\partial n}=-\frac{\partial \phi}{\partial y}=0 & \text { at } y=h
\end{array}
$$

where $D / D t$ in ( 2 ) denotes the substantial derivative; $\dot{y}_{0}(t)$ in ( 3$)$ is the velocity of the wavemaker, and $\boldsymbol{n}=$ $\left(n_{1}, n_{2}\right)$ is the unit normal vector; $n_{3}$ in $(4)$ is defined as $n_{3}=\left(x-x_{G}-\xi_{1}\right) n_{2}-\left(y-y_{G}-\xi_{2}\right) n_{1}$, and $v_{1}=\dot{\xi}_{1}(t), v_{2}$ $=\dot{\xi}_{2}(t)$, and $v_{3}=\dot{\alpha}(t)$. Note that the normal vector is defined in the spaced-fixed coordinate system and positive when directing from the body surface into the fluid.

The far-field condition of outgoing waves is satisfied by installing an artificial wave absorbing beach. Mathematically, as in the previous paper ${ }^{5)}$ for the radiation problem, Newtonian cooling terms are introduced only in the kinematic free-surface condition in (2), in the form

$$
\frac{D x}{D t}=\frac{\partial \phi}{\partial x}, \frac{D y}{D t}=\frac{\partial \phi}{\partial y}-2 \nu y-\nu^{2} \phi \quad \text { on } S_{W}
$$

Here $\nu$ is nonzero inside the beach and given by

$$
\nu=3 C_{s}\left(x-x_{f}\right)^{2} / C_{W}^{3} \text { for } x \geq x_{f}
$$

with $x_{f}$ being the starting point of the wave absorbing beach which extends over a length $C_{W}$ (see Fig. 1). The value of coefficient $C_{S}$ is taken equal to 1.2 for all computations in this paper.

\section{Acceleration Field and Force}

To compute the velocity field at some time instant, the displacement and velocity of a floating body must be known; these may be given by integrating in time the equations of the body motion expressed in the form

$$
m a_{1}=F_{1}, m a_{2}=F_{2}, m \kappa^{2} a_{3}=F_{3}
$$

where $m$ and $\kappa$ are the body mass and the gyrational radius in roll, respectively, and $a_{1}=\ddot{\xi}_{1}(t), a_{2}=\ddot{\xi}_{2}(t)$, and $a_{3}=\ddot{\alpha}(t)$ are the components of the acceleration defined in the space-fixed reference frame.

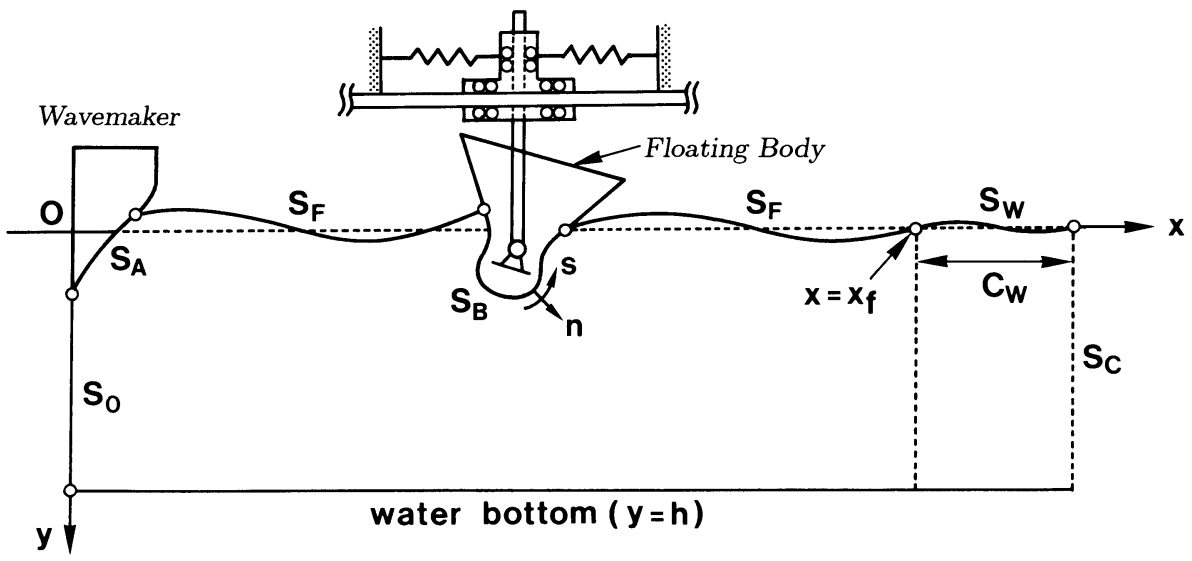

Fig. 1 Coordinate system and schematic view of experiment 
The right-hand side of each equation in $(9), F_{i}$, is the force $(i=1,2)$ and moment $(i=3)$ on a body, in which the components of hydrodynamic and hydrostatic forces may be obtained by integrating Bernoulli's pressure over the wetted surface of a body. Including the gravitational force and horizontal restoring force due to a weak spring, the total force in the $i$-th direction can be computed by

$$
F_{i}=\int_{S_{B}}\left\{\phi_{t}+\frac{1}{2} \nabla \phi D \phi-y\right\} n_{i} d \ell-k \xi_{1} \delta_{i 1}+W \delta_{i 2}
$$

where $\phi_{t}=\partial \phi / \partial t$ is the temporal derivative of the velocity potential, $k$ is the linear spring constant, and $W$ is the weight of the body, equal to $m g$. $\delta_{i j}$ denotes the Kronecker delta, equal to 1 when $i=j$, otherwise zero.

A numerical problem in (10) is evaluating $\phi_{t}$. The simplest way for that may be to use a backward finitedifference scheme in time. However, it has been shown that the use of a backward finite-difference scheme makes a solution unstable, resulting in the breakdown of computations. Several recent works, e. g. Cointe et al. $^{8)}$ and Tanizawa ${ }^{3)}$, recommend to solve the boundaryvalue problem for $\phi_{t}$ for stable and accurate simulations of motions of a freely oscillating body.

According to Tanizawa ${ }^{3)}$, the body boundary condition for $\phi_{t}$ can be written as

$$
\frac{\partial \phi_{t}}{\partial n}=\sum_{j=1}^{3} a_{j} n_{j}+q_{B} \quad \text { on } S_{B}
$$

where

$$
\begin{aligned}
q_{B}= & -k_{n}\left\{\left(\frac{\partial \phi}{\partial x}-\dot{\xi}_{1}+\dot{\alpha}\left(y-y_{G}-\xi_{2}\right)\right)^{2}\right. \\
& \left.+\left(\frac{\partial \phi}{\partial y}-\dot{\xi}_{2}-\dot{\alpha}\left(x-x_{G}-\xi_{1}\right)\right)^{2}\right\} \\
& +n_{1}\left\{\dot{\alpha}^{2}\left(x-x_{G}-\xi_{1}\right)-2 \dot{\alpha}\left(\frac{\partial \phi}{\partial y}-\dot{\xi}_{2}\right)\right\} \\
& +n_{2}\left\{\dot{\alpha}^{2}\left(y-y_{G}-\xi_{2}\right)+2 \dot{\alpha}\left(\frac{\partial \phi}{\partial x}-\dot{\xi}_{1}\right)\right\} \\
& +k_{n}\left\{\left(\frac{\partial \phi}{\partial x}\right)^{2}+\left(\frac{\partial \phi}{\partial y}\right)^{2}\right\} \\
& +\frac{\partial \phi}{\partial n} \frac{\partial}{\partial s}\left(\frac{\partial \phi}{\partial s}\right)-\frac{\partial \phi}{\partial s} \frac{\partial}{\partial s}\left(\frac{\partial \phi}{\partial n}\right)
\end{aligned}
$$

Here $k_{n}$ is the curvature of body surface, and $s$ is the tangential direction orthogonal to $n$ (see Fig.1).

It should be noted that $q_{B}$ defined by (12) can be explicitly evaluated from solutions of the velocity field, whereas the components of the body acceleration, $a_{j}$, are unknown and to be given from (9) and (10). Namely, the calculation of the acceleration field is coupled with the body-motion equations.

The boundary condition on the wavemaker $\left(S_{A}\right)$ can be obtained simply by substituting $\xi_{1}=\alpha=0$ and $\xi_{2}=$ $y_{0}(t)$ into (11), with the result

$$
\frac{\partial \phi_{t}}{\partial n}=q_{A} \quad \text { on } S_{A}
$$

where

$$
\begin{aligned}
q_{A}= & \ddot{y}_{0} n_{2}-k_{n} \dot{y}_{0}\left(\dot{y}_{0}-2 \frac{\partial \phi}{\partial y}\right) \\
& +\frac{\partial \phi}{\partial n} \frac{\partial}{\partial s}\left(\frac{\partial \phi}{\partial s}\right)-\frac{\partial \phi}{\partial s} \frac{\partial}{\partial s}\left(\frac{\partial \phi}{\partial n}\right)
\end{aligned}
$$

Since $y_{0}(t)$ may be given as an input, the right-hand side of (13), $q_{A}$, can be explicitly evaluated.

Other boundary conditions for $\phi_{t}$ are written as follows :

$$
\begin{array}{ll}
\phi_{t}=-\frac{1}{2} \nabla \phi D \phi+y & \text { on } S_{F} \\
\frac{\partial \phi_{t}}{\partial n}=\frac{\partial \phi_{t}}{\partial x}=0 & \text { on } S_{0} \\
\frac{\partial \phi_{t}}{\partial n}=-\frac{\partial \phi_{t}}{\partial y}=0 & \text { at } y=h
\end{array}
$$

Eq. (15) has been obtained from the dynamic free-surface condition of $p=0$.

The above boundary-value problem for $\phi_{t}$ is the same in nature as that for $\phi$ itself. Therefore, as will be explained later, the velocity and acceleration fields can be calculated with the same numerical scheme based on the boundary-element method. However, it should be noted that the right-hand sides of (12)-(15) may not be evaluated unless the velocity field is completely determined. This implies that the boundaryvalue problem for $\phi$ and $\phi_{t}$ can not be solved at the same time.

\section{Mode Decomposition Method}

Several methods have been proposed for solving the acceleration field coupled with the motion equations of a floating-body; for instance, the iteration method by Cao et al. $^{2}$, the mode decomposition method by Vinje \& Brevig $^{7)}$ and Cointe et $\mathrm{al}^{8}{ }^{8}$, and the implicit boundarycondition method by Tanizawa ${ }^{3)}$. These are essentially the same in that the boundary-value problem for $\phi_{t}$ must be solved after the velocity field is completely determined. In this section, the mode decomposition method will be summarized as a typical conventional method.

Since the body boundary condition (11) is linear with respect to the acceleration, $\phi_{t}$ may be obtained in the form

$$
\phi_{t}=\sum_{j=1}^{3} a_{j} \psi_{j}+\phi_{4}
$$

Then, $\psi_{j}$ must be calculated so as to satisfy the followings :

$$
\begin{aligned}
& \frac{\partial \psi_{j}}{\partial n}=\left\{\begin{array}{ll}
n_{j}(j=1 \sim 3) \\
q_{B}(j=4)
\end{array} \text { on } S_{B}\right. \\
& \frac{\partial \psi_{j}}{\partial n}= \begin{cases}0 & (j=1 \sim 3) \\
q_{A}(j=4) & \text { on } S_{A}\end{cases} \\
& \psi_{j}=\left\{\begin{array}{ll}
0 & (j=1 \sim 3) \\
-\frac{1}{2} \nabla \phi D \phi+y & (j=4)
\end{array} \text { on } S_{F}\right. \\
& \frac{\partial \psi_{j}}{\partial n}=0 \quad \text { on } S_{0} \text { and at } y=h
\end{aligned}
$$

With this decomposition, $\psi_{j}$ can be determined irrespective of the body acceleration, and the boundaryvalue problems for $j=1 \sim 3$ are independent of the velocity field. It is noteworthy that (18) and (19) are reminiscent of the decomposition in the linear theory into the radiation and diffraction problems.

Once the boundary-value problems for $\psi_{j}$ are solved, 
the total force in the $i$-th direction can be obtained in the form

$$
F_{i}=-\sum_{j=1}^{3} a_{j} A_{i j}+Q_{i}
$$

where

$$
\begin{aligned}
& A_{i j}=-\int_{S_{B}} \psi_{j} n_{i} d \ell \\
& Q_{i}=\int_{S_{B}}\left\{\psi_{4}+\frac{1}{2} \nabla \phi D \phi-y\right\} n_{i} d \ell-k \xi_{1} \delta_{i 1}+W \delta_{i 2}(25)
\end{aligned}
$$

Here $A_{i j}$ may be understood as the added mass and $Q_{i}$ represents the other forces, dependent on the velocity, displacement, and incident waves.

Substituting (23) in (9), the components of the body acceleration can be explicitly given, which will give the velocity and displacement of the body in the next time step.

\section{New Indirect Method}

One defect in the above method is that the $\psi_{4}$-problem can not be solved unless the velocity field is completely determined. That is, after solving the boundary-value problems for $\phi$ and $\phi_{j}(j=1 \sim 3)$, the boundary-value problem for $\psi_{4}$ must be solved separately in one time step; which is disadvantageous from a viewpoint of computation time, especially for long time simulations.

A favorable style in that respect is that the boundaryvalue problems for the velocity and acceleration fields can be solved at the same time. This requirement may be achieved by an idea proposed by Wu \& Eatock Taylor ${ }^{6}$. Unfortunately, the body boundary condition for $\phi_{t}$ in their paper is different from (11) and no numerical results have been published. Therefore, necessary equations for computing the acceleration of a body, particularly the modification of $Q_{i}$ given by (25), will be described below.

In $\mathrm{Wu} \&$ Eatock Taylor ${ }^{6)}$, Green's second identity was applied to $\phi_{t}$ and $\phi_{i}(i=1 \sim 3)$, but here we consider that identity for $\psi_{4}$ and $\psi_{i}(i=1 \sim 3)$, which is written as

$$
\int_{S_{0}+S_{A}+S_{B}+S_{F}+S_{\infty}}\left\{\psi_{4} \frac{\partial \psi_{i}}{\partial n}-\psi_{i} \frac{\partial \psi_{4}}{\partial n}\right\} d \ell=0
$$

From the boundary conditions to be satisfied by $\psi_{4}$ and $\phi_{i}$, the integral along $S_{0}$ and $S_{\infty}$ (which includes $S_{C}$ and the water bottom) may be zero. Further, taking account of $\psi_{i}=0$ on $S_{F}, \partial \psi_{i} / \partial n=0$ on $S_{A}$, and $\partial \psi_{i} / \partial n=n_{i}$ on $S_{B}$, it follows that

$$
\begin{aligned}
\int_{S_{B}} \psi_{4} n_{i} d \ell= & \int_{S_{A}+S_{B}} \psi_{i} \frac{\partial \psi_{4}}{\partial n} d \ell-\int_{S_{F}} \psi_{4} \frac{\partial \psi_{i}}{\partial n} d \ell \\
= & \int_{S_{A}} \psi_{i} q_{A} d \ell+\int_{S_{B}} \psi_{i} q_{B} d \ell \\
& +\int_{S_{F}}\left\{\frac{1}{2} \nabla \phi D \phi-y\right\} \frac{\partial \psi_{i}}{\partial n} d \ell
\end{aligned}
$$

In obtaining (27), conditions of (19)-(21) have been used.

With (27), the force term $Q_{i}$ defined by (25), can be calculated as follows:

$$
\begin{aligned}
Q_{i}= & \int_{S_{A}} \psi_{i} q_{A} d \ell+\int_{S_{B}} \psi_{i} q_{B} d \ell \\
& +\int_{S_{B}+S_{F}}\left\{\frac{1}{2} D \phi D \phi-y\right\} \frac{\partial \psi_{i}}{\partial n} d \ell-k \xi_{1} \delta_{i 1}+W \delta_{i 2}
\end{aligned}
$$

It should be emphasized that there is no need to solve the $\psi_{4}$-problem for computing (28) and that the boundary-value problems for $\psi_{i}(i=1 \sim 3)$ and $\phi$ are independent and thus can be solved at the same time. With these facts, we can expect reduction of the computation time compared to the mode decomposition method.

We note that transformations in this new method are reminiscent of a proof of the Haskind relation in the linear theory, saying that the wave-exciting force can be computed in terms of only the radiation solutions. Another thing to be noted is that the pressure is not calculated in the new method and hence a solution of the $\psi_{4}$-problem is still needed if the pressure distribution is of concern.

\section{Numerical Procedure}

In the MEL method, the positions of fluid particles ( $x$, $y$ ) on the free surface and the velocity potential $\phi$ on those points are pursued by integrating (2) in time. This means that the boundary condition for $\phi$ on $S_{F}$ is of Dirichlet type, which is the same as that for $\psi_{j}$, (21).

Therefore, at each time step, the velocity field described by $\phi$ and the artificial flow described by $\psi_{j}$ can be determined in the same manner. Let $\phi$ or $\psi_{j}$ be denoted by $\psi$ in general. Then a solution of $\psi$ can be obtained by solving the boundary integral equation of the form

$$
\begin{aligned}
& C(P) \psi(P)+\int_{S_{0}+S_{A}+S_{B}} \psi(Q) \frac{\partial G(P ; Q)}{\partial n_{Q}} d \ell \\
&-\int_{S_{F}+S_{W}} \frac{\partial \psi(Q)}{\partial n_{Q}} G(P ; Q) d \ell \\
&=\int_{S_{A}+S_{B}} \frac{\partial \psi(Q)}{\partial n_{Q}} G(P ; Q) d \ell \\
&-\int_{S_{F}+S_{W}} \psi(Q) \frac{\partial G(P ; Q)}{\partial n_{Q}} d \ell
\end{aligned}
$$

where

$$
\left.\begin{array}{l}
G(P ; Q)=\frac{1}{2 \pi}\left(\log r+\log r_{h}\right) \\
r=\sqrt{\left(x-x^{\prime}\right)^{2}+\left(y-y^{\prime}\right)^{2}} \\
r_{h}=\sqrt{\left(x-x^{\prime}\right)^{2}+\left\{y-\left(2 h-y^{\prime}\right)\right\}^{2}}
\end{array}\right\}
$$

$P=(x, y)$ is a point on the boundary and $Q=\left(x^{\prime}, y^{\prime}\right)$ is an integration point. $C(P)$ is referred to as the solid angle, which is computed numerically from the equipotential condition over the entire boundary.

As shown in (30), the Green function includes the mirror image reflected in the water bottom, so that the water bottom is excluded from the integration area in (29). Also excluded is a control surface $S_{c}$ shown in Fig. 1, because $\psi=0$ and $\partial \psi / \partial n=0$ are expected by virtue of the absorbing beach.

To solve (29) accurately, a higher-order boundaryelement method is applied using quadratic isoparametric elements, which is the same as in the previous paper ${ }^{5}$. Once the values at nodes are determined, higher-order shape functions may be used for the interpolation; in this paper the Lagrangian interpolation function is used for that purpose.

In a higher-order boundary-element method, a double 
node is placed at the intersection between the free surface and a freely floating body. Then the potential $\psi$ at a double node is assumed to be single-valued, but $\partial \psi / \partial n$ on $S_{F}$ and $S_{B}$ can be different. In the present case, $\psi$ on $S_{F}$ is given from the Dirichlet condition, ( 2 ) and (21), and $\partial \psi / \partial n$ on $S_{B}$ is specified by the body boundary conditions, (4) and (19). Therefore the only unknown at the intersection is $\partial \psi / \partial n$ on $S_{F}$, which will be obtained as a solution of (29). The same numerical treatment is used at the intersection between the free surface and the wavemaker.

The 4-th order Runge-Kutta-Gill method is adopted as the time-marching scheme. At each time step, rearranging the nodes on the free surface is conducted to avoid dense or sparse distribution of nodes, which is necessary for stable and accurate simulations for a long time.

\section{Results and Discussion}

\section{1 Tested models and experiments}

Experiments were carried out in the wave channel (length $\times$ breadth $\times$ depth $=14 \mathrm{~m} \times 0.30 \mathrm{~m} \times 0.45 \mathrm{~m}$ ) at Osaka University. As shown schematically in Fig. 1, a plunger-type wavemaker is installed at the left end of the wave channel. The section shape and principal dimensions of a float of the wavemaker are shown in Fig. 2.

Two different floating bodies were used in experiments; one is a wall-sided model shown in Fig. 3, similar to the shape of midship section of a ship, and the other is a flared model shown in Fig. 4, similar to the shape of bow section of a ship. Other data associated with the equations of the body motion are listed in Table 1 . Both models were initially placed at $x_{G}=3.586$ $\mathrm{m}$ from the rear of the wavemaker, i. e. the origin of the coordinate system.

Measured items are the motions (sway, heave, and

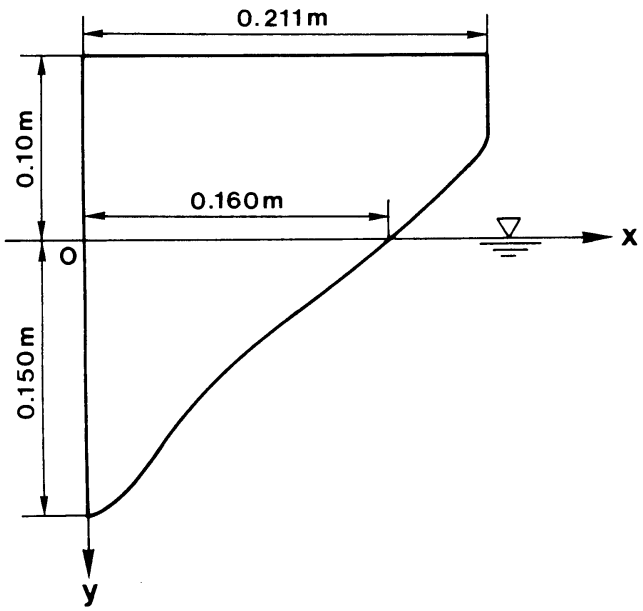

Fig. 2 Section shape and principal dimensions of the wavemaker

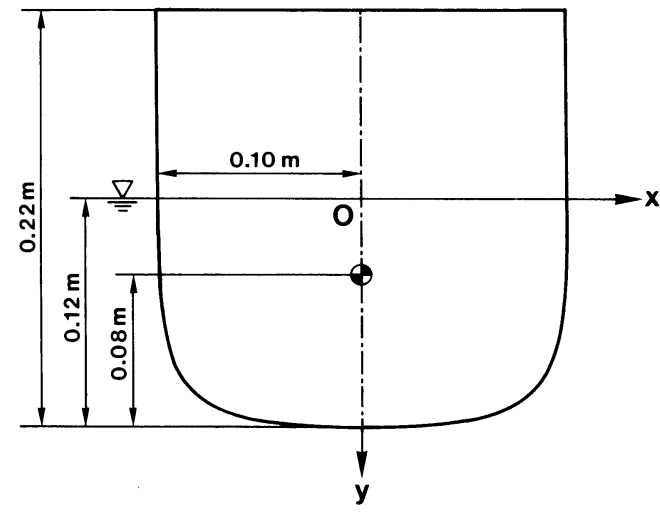

Fig. 3 Section shape and principal dimensions of a wall-sided model

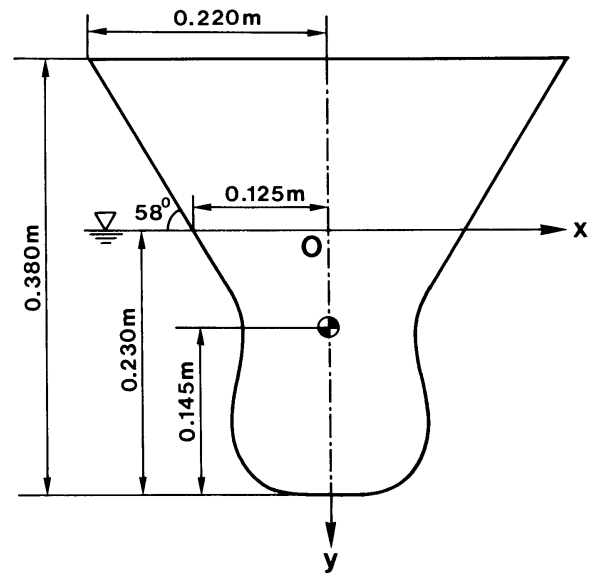

Fig. 4 Section shape and principal dimensions of a flared model

Table 1 Principal dimensions of tested models and constants in experiments

\begin{tabular}{|ll|c|c|}
\hline Kind of tested model & & Wall-sided & Flared \\
\hline \hline Half breadth at W.L. & $(b)$ & $0.100 \mathrm{~m}$ & $0.125 \mathrm{~m}$ \\
Draft & $(d)$ & $0.120 \mathrm{~m}$ & $0.230 \mathrm{~m}$ \\
Length & $(L)$ & $0.297 \mathrm{~m}$ & $0.297 \mathrm{~m}$ \\
Center of gravity & $\left(y_{G}\right)$ & $0.040 \mathrm{~m}$ & $0.085 \mathrm{~m}$ \\
Radius of gyration & $(\kappa)$ & $0.067 \mathrm{~m}$ & $0.123 \mathrm{~m}$ \\
Natural period of heave & $\left(T_{h}\right)$ & $0.82 \mathrm{sec}$ & $0.93 \mathrm{sec}$ \\
Natural period of roll & $\left(T_{r}\right)$ & $1.07 \mathrm{sec}$ & $2.25 \mathrm{sec}$ \\
\hline Spring constant & $(k)$ & \multicolumn{2}{|c|}{$42.44 \mathrm{~N} / \mathrm{m}$} \\
Weight of swaying carriage & & \multicolumn{2}{|c|}{$6.272 \mathrm{~N}$} \\
\hline
\end{tabular}

roll) of a floating body and the wave elevations at $x=$ $1.940 \mathrm{~m}$ (between the wavemaker and a floating body) and at $x=4.676 \mathrm{~m}$ (the downwave side of a floating body). The movement of the wavemaker from the state of rest was also measured, which was found to include higher harmonic terms and thus approximated with a mathematical function of the form 


$$
\begin{gathered}
y_{0}(t)=M(t)\left(a_{0}+b_{1} \sin \omega t+b_{2} \sin 2 \omega t\right. \\
\left.\quad+a_{2} \cos 2 \omega t\right) \\
M(t)=1-e^{-\alpha t-\beta t^{2}}
\end{gathered}
$$

Coefficients $\alpha, \beta, a_{0}, b_{1}, b_{2}$ and $a_{2}$ were determined for each run of experiments by the least-square method using the iteration.

\section{2 Waves generated by the wavemaker}

When a floating body is absent, the present calculation scheme is the same as in the previous paper ${ }^{5)}$, in which good agreement was confirmed between calculated and measured waves generated by a plunger-type wavemaker with wedge section. However, the experiments in this paper were carried out in a wave channel of small size and the section shape of the wavemaker is different from the previous one. Therefore, as a check of input waves impinging upon a floating body, simulations are performed first only for the waves generated by the wavemaker.

Two examples are shown here; Fig. 5 is the results for the oscillation period $T=0.9 \mathrm{sec}$ and the wave steepness (the ratio of wave height to wavelength) $H / \lambda \approx$ 0.022 , and Fig. 6 is a case of longer wave with $T=1.2$ sec and $H / \lambda \approx 0.019$. Results are shown in nondimensional form in terms of the oscillation amplitude of the wavemaker, $Y$. The abscissa is the nondimensional time $t \sqrt{g / a}$, with $a=0.160 \mathrm{~m}$ being the breadth of the wavemaker at water line. Each figure is plotted such that the positive of the ordinate indicates the upward direction of movement.

In numerical computations, the length of the wave absorbing beach, $C_{W}$, is taken equal to $2 \lambda$, and the time ${ }^{-}$
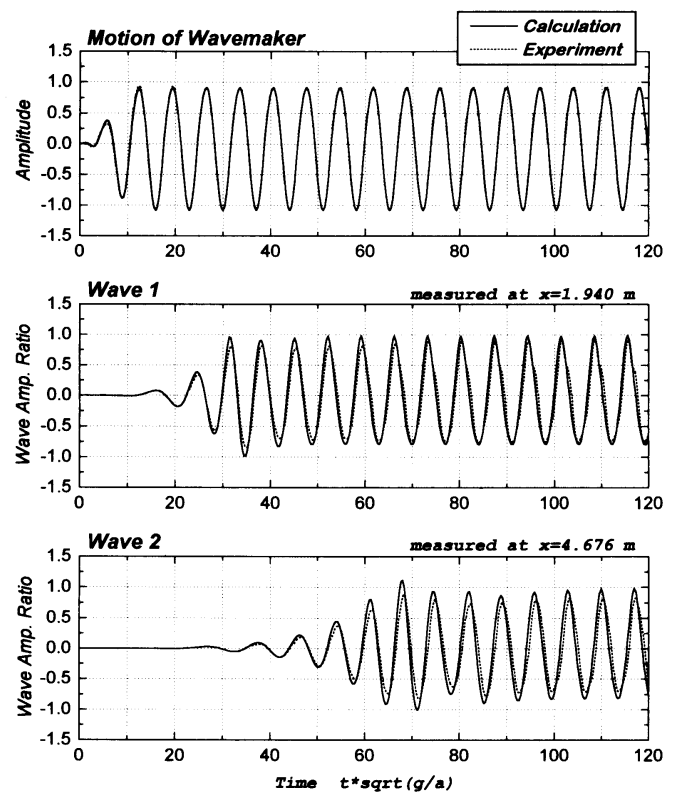

Fig. 5 Surface waves generated by the wavemaker shown in Fig. 2. The oscillation period is $T=0.9$ sec and the wave steepness is $H / \lambda \approx 0.022$. step size $\Delta t$ is selected to be $T / 20$, with $T$ the oscillation period. The number of discretized elements is 30 on the wavemaker and 30 per wavelength on the free surface.

The overall agreement is very good, but we can point out two things. Firstly, measured waves include some disturbances, because the wave profile becomes distorted from that to be expected as a nonlinear wave, as the time elapses. Secondly, in a case of shorter wave, the wave amplitude is slightly attenuating with increasing the distance from the wavemaker; this may be due to a viscous effect from the boundary layer on side walls of the wave channel.

\section{3 Floating-body motions in waves}

When a floating body is placed on the free surface, the pursuit of the intersection between the body and free surfaces is crucial and the numerical accuracy of $q_{B}$ expressed by (12) may influence subsequent results in the time marching. For these reasons, the number of discretized elements on the wetted surface of a floating body must be relatively large. In the present computations, 80 elements are used on $S_{B}$. The number of elements on other boundaries can be the same as in the preceding problem without a floating body. The length of the wave absorbing beach, $C_{w}$, and the time-stepping size, $\Delta t$, are also the same; that is, $C_{W}=2 \lambda$ and $\Delta t=$ $T / 20$.

It should be mentioned firstly that the numerical results by the new indirect method described in Section 5 are substantially the same as those by the mode decomposition method described in Section 4. The
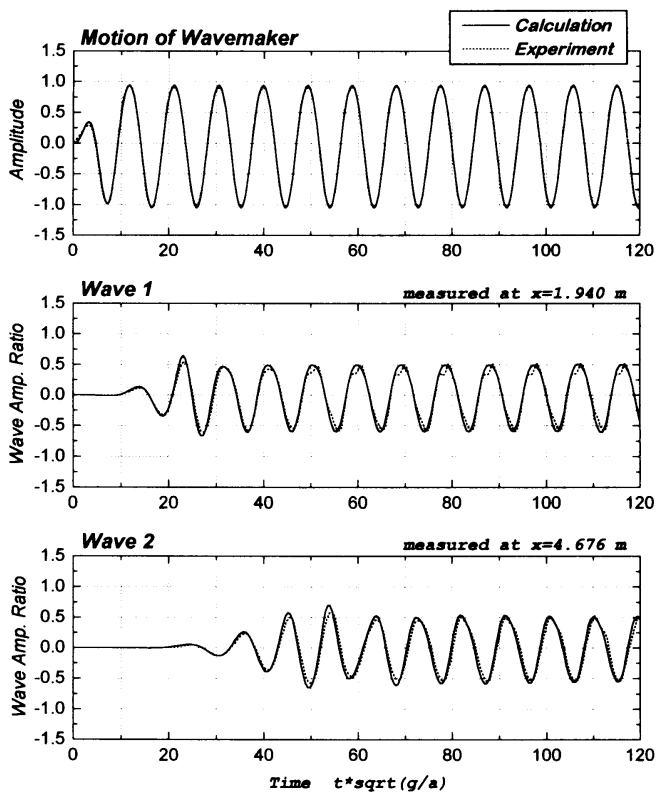

Fig. 6 Surface waves generated by the wavemaker shown in Fig. 2. The oscillation period is $T=1.2$ sec and the wave steepness is $H / \lambda \approx 0.0185$. 
difference may not be distinguished in figures which will be shown below. This means that Eq. (26) is satisfied within a very small error, because the mathematical difference between the two methods stems from applying (26). However, we emphasize that the computation time in the new method is considerably reduced and roughly half of the computation time in the modedecomposition method.

The results for the wall-sided model are shown in Figs. 7 and 8 in nondimensional form. The oscillation period of the wavemaker in Fig. 7 is $0.85 \mathrm{sec}$, which is close to the natural period of heave $\left(T_{h}=0.82 \mathrm{sec}\right)$. Hence the heave amplitude is certainly large. However, measured results of heave are smaller than the calculated results. This difference can be partly explained from that the present calculation is based on the potential theory. Another reason is that the measured incident wave, the input, is slightly smaller than the calculated one, which has been already pointed out in relation to Fig. 5.

In the present computations and experiments, the reflection of waves is repeated between a floating body and the wavemaker. This is evident from the time history of the wave elevation labeled as Wave 1. Although there are some small differences in the amplitude, the overall agreement is very good.

Fig. 8 is an example of a longer-wave case and the oscillation period is $T=1.1 \mathrm{sec}$. Since this period is close to the natural period of roll $\left(T_{r}=1.07 \mathrm{sec}\right)$, the amplitude of roll is much larger than that in Fig. 7. Here again measured results are smaller than the calculated results, which may be due to viscous effects. The
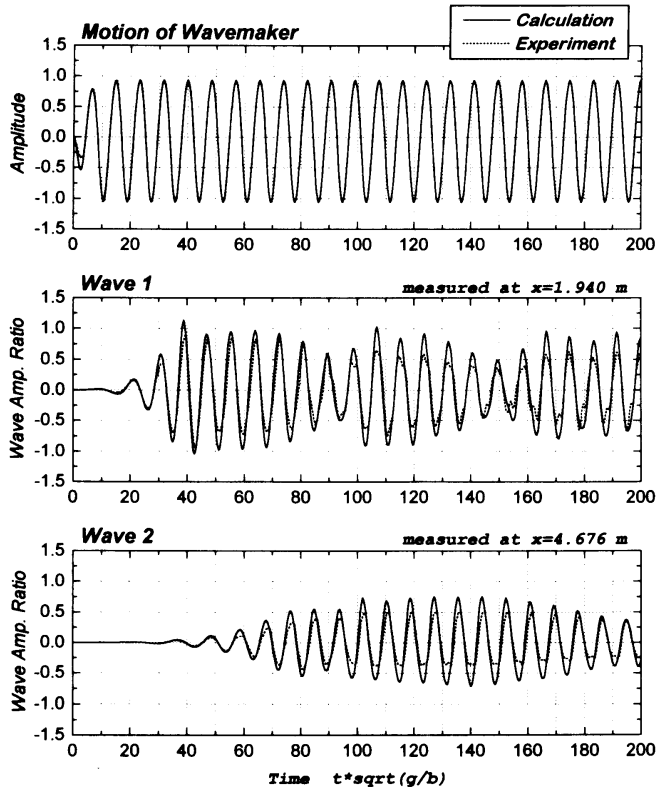

waves are in good agreement, including a distorted profile after wave reflections. It can be seen for the wallsided model that the oscillation period of roll is the same as that of the incident wave and the interaction between heave and roll is not large.

Next comparisons are for the flared model. Fig. 9 is the results for the oscillation period of $T=0.95 \mathrm{sec}$ and the wave steepness of approximately $H / \lambda=0.02$. This oscillation period is selected close to the natural period of heave $\left(T_{h}=0.930 \mathrm{sec}\right)$.

Similar to the case of wall-sided model, the amplitudes of computed heave and sway are slightly larger than the measurements, but the phases are in good agreement. A big difference from the wall-sided model exists in the roll motion. We can see the steady inclination in the beginning of motion and then transition to a large amplitude of the parametric oscillation with the period of twice the heave oscillation period. This parametric oscillation may be induced by a reflected wave from the wavemaker (the amplitude of which is increased as seen from the time history of Wave 1). These transient and nonlinear phenomena are well accounted for by the present calculations.

It is known that the parametric oscillation in roll is most likely to occur when the period of the incident wave is close to half of the natural period of roll. Fig. 10 is the results for $T=1.2 \mathrm{sec}$ and $H / \lambda \approx 0.013$, and as shown in Table 1 , the roll natural period of the flared model is $T_{r}=2.25 \mathrm{sec}$.

Despite a small amplitude of the incident wave, the parametric oscillation is induced after transition for a certain period of time. Tanizawa et al. ${ }^{9)}$ studied the
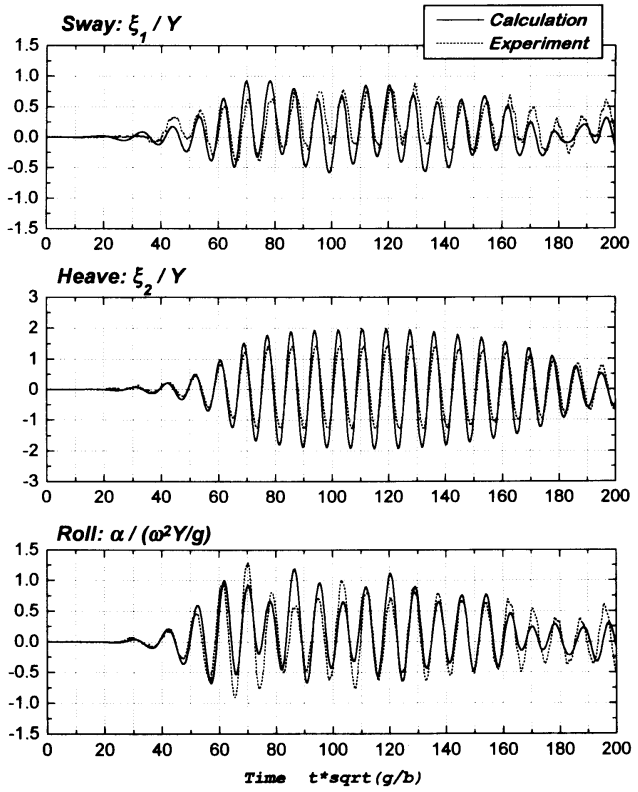

Fig. 7 Time histories of wave elevations, sway, heave, and roll of the wall-sided model shown in Fig. 3. The oscillation period is $T=0.85 \mathrm{sec}$ and the wave steepness is $H / \lambda \approx 0.0129$. 

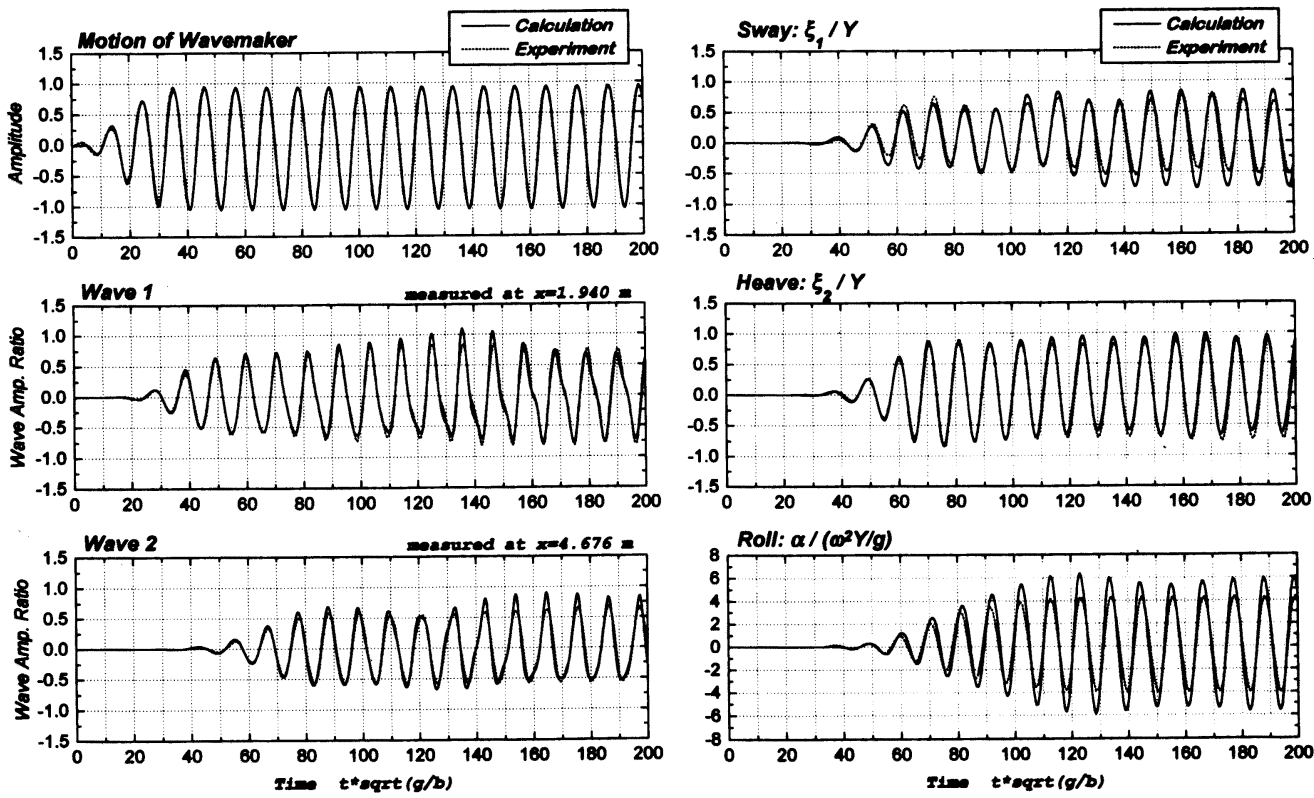

Fig. 8 Time histories of wave elevations, sway, heave, and roll of the wall-sided model shown in Fig. 3. The oscillation period is $T=1.10 \mathrm{sec}$ and the wave steepness is $H / \lambda \approx 0.0235$.
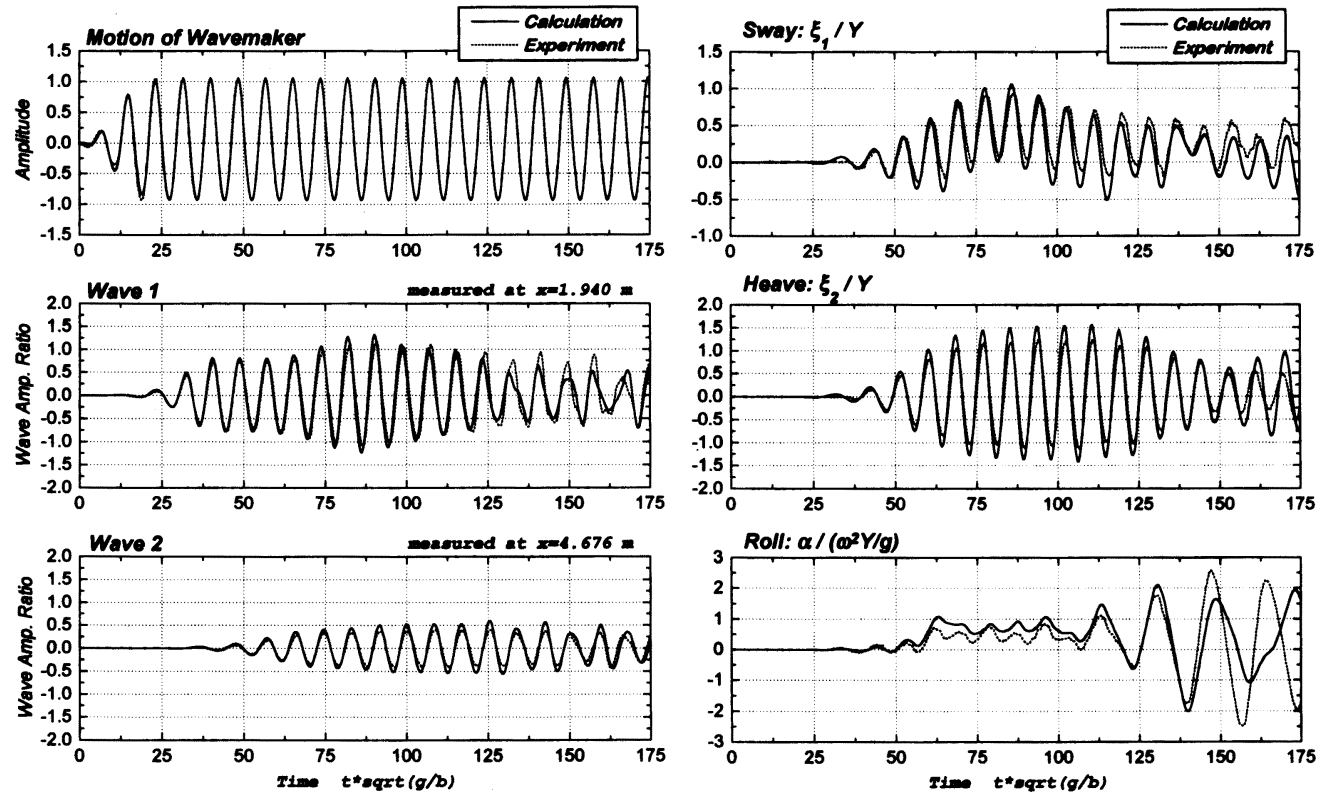

Fig. 9 Time histories of wave elevations, sway, heave, and roll of the flared model shown in Fig. 4 . The oscillation period is $T=0.95 \mathrm{sec}$ and the wave steepness is $H / \lambda \approx 0.0199$.

amplitude dependence on the parametric oscillation. According to that study, we can expect that the inception of the parametric oscillation becomes earlier as the amplitude of the incident wave increases. The correctness of this speculation is supported by Fig. 11, in which the oscillation period remains the same as Fig. 10, whereas the wave steepness is increased to $H / \lambda \approx 0.0184$. (In Fig. 11, the measurement was intentionally stopped at $t \sqrt{g / b} \approx 93$, because the roll amplitude approached the mechanical limit angle for measurement.) At any rate, in both cases of Figs. 10 and 11, calculated results are in excellent agreement with measured results. 

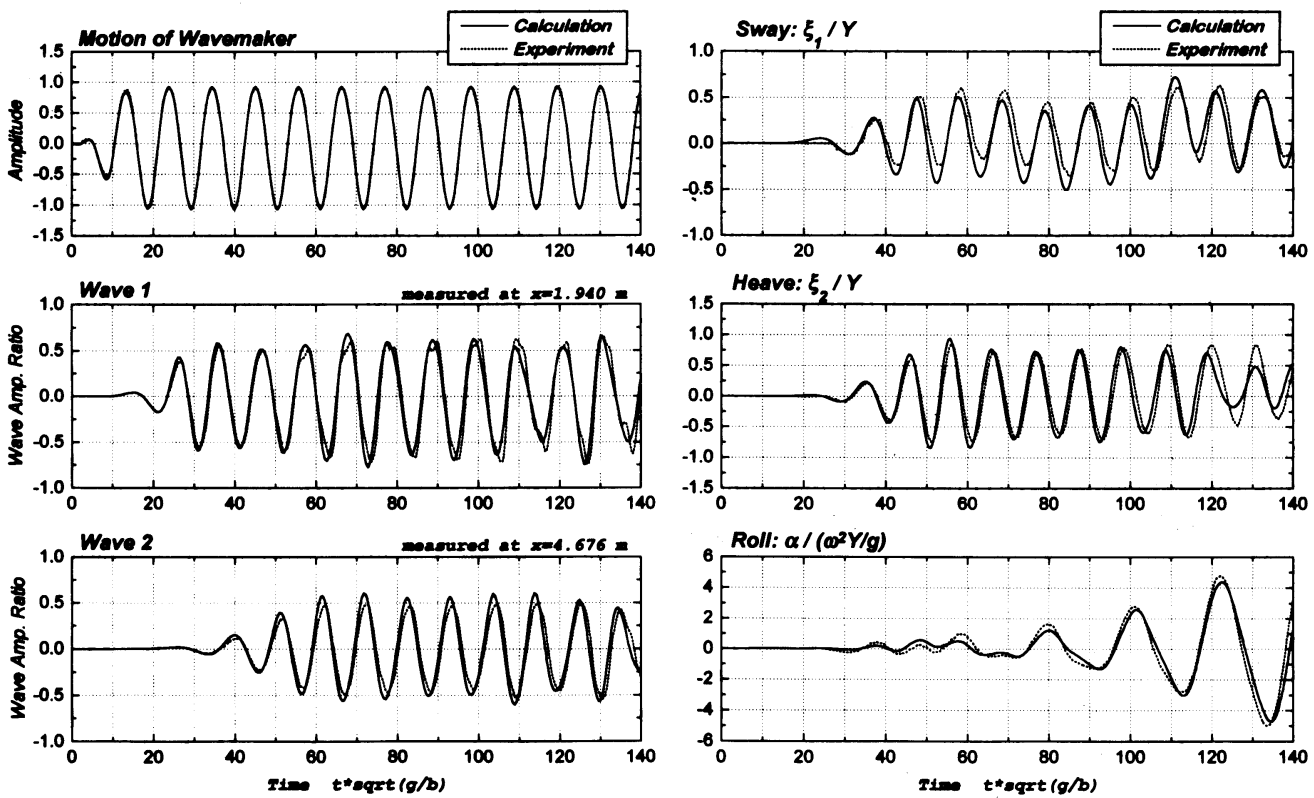

Fig. 10 Time histories of wave elevations, sway, heave, and roll of the flared model shown in Fig. 4. The oscillation period is $T=1.20 \mathrm{sec}$ and the wave steepness is $H / \lambda \approx 0.0132$.
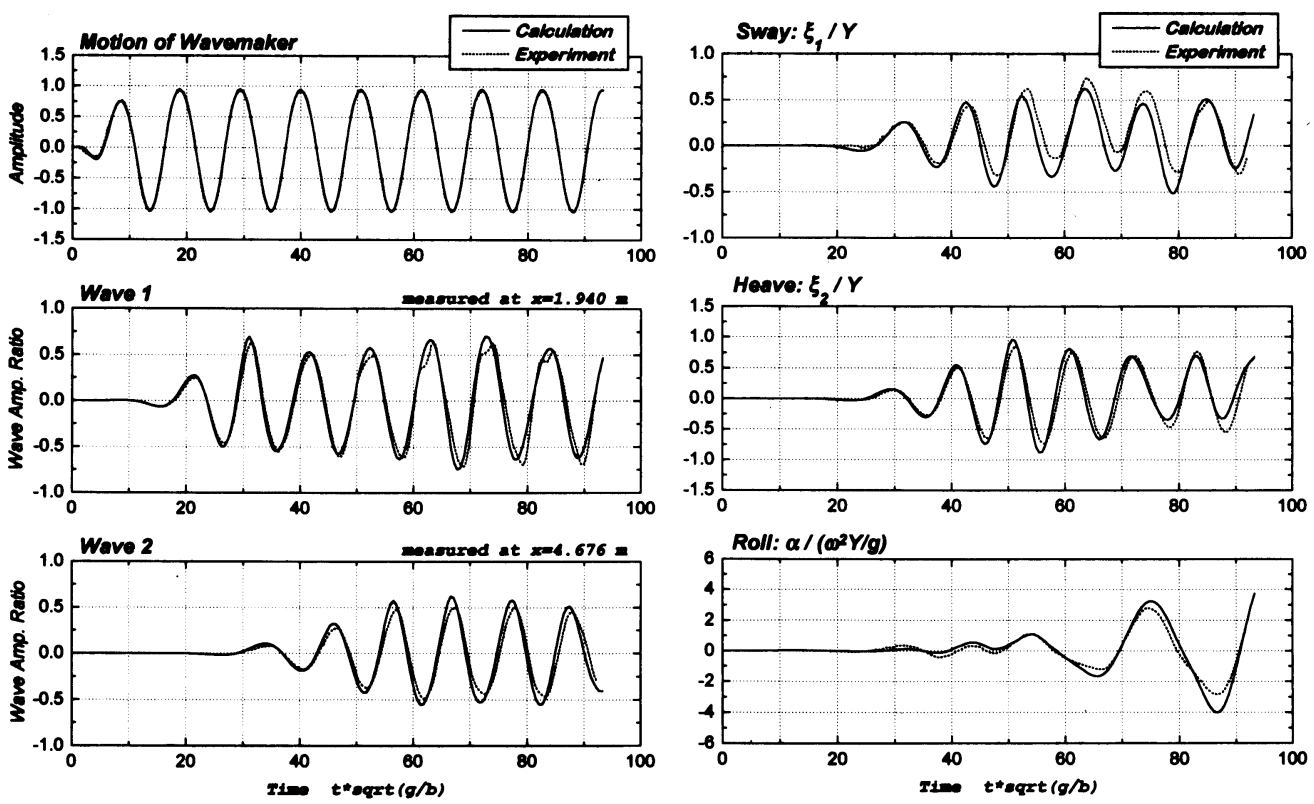

Fig. 11 Time histories of wave elevations, sway, heave, and roll of the flared model shown in Fig. 4. The oscillation period is $T=1.20 \mathrm{sec}$ and the wave steepness is $H / \lambda \approx 0.0184$.

\section{Concluding Remarks}

A nonlinear simulation method was presented for wave-induced transient motions of a floating body, using a new indirect method to compute the hydrodynamic force on and the acceleration of a body.
With Green's second identity, the new method was explained as a further extension from the mode decomposition method. It is interesting to note that mathematical transformations in the new method are reminiscent of a derivation of the Haskind relation in the linear theory. 
It was confirmed that the results by the new method are in virtually perfect agreement with the ones by the mode decomposition method. However, the new method is greatly advantageous in reducing the computation time, because there is no need to solve the boundary-value problem for $\phi_{t}$ itself. Conversely, the new method has a disadvantage, not capable of providing the pressure distribution.

Of course, the MEL method has a fatal defect that the computation cannot proceed once the wave breaking occurs. However, as Tanizawa et al. ${ }^{9,10)}$ has shown recently, we believe that the MEL method is still useful in studying nonlinear phenomena. Future work in this direction is the extension to $3-\mathrm{D}$ problems, including a ship-motion problem with forward speed in severe waves.

The authors would like to thank Prof. Naito of Osaka University who allowed us to use the wave channel in his laboratory for carrying out the experiments shown in this paper.

\section{References}

1) Longuet-Higgins, M. S. and Cokelet, E.: The Deformation of Steep Surface Waves on Water, Proc. Roy. Soc. London, Series A 350 (1976). pp. $1-26$

2) Cao, Y., Beck, R. and Schultz, W. W. : Nonlinear Motions of Floating Bodies in Incident Waves, Proc. 9th Int. Workshop on Water Waves and
Floating Bodies, Oita (1994), pp. 33-37.

3) Tanizawa, K.: A Nonlinear Simulation Method of 3-D Body Motions in Waves (1st Report), J. Soc. Nav. Arch. Japan, Vol. 178 (1995), pp. 179191.

4) Tanizawa, K. : Long Time Fully Nonlinear Simulation of Floating Body Motions with Artificial Damping Zone, J. Soc. Nav. Arch. Japan, Vol. 180 (1996), pp. 311-319.

5) Kashiwagi, M.: Full-Nonlinear Simulations of Hydrodynamic Forces on a Heaving TwoDimensional Body, J. Soc. Nav. Arch. Japan, Vol. 180 (1996), pp. 373-381.

6) $\mathrm{Wu}, \mathrm{G} . \mathrm{X}$. and Eatock Taylor, R.: Transient Motion of a Floating Body in Steep Water Waves, Proc. 11th Int. Workshop on Water Waves and Floating Bodies, Hamburg (1996).

7) Vinje, T. and Brevig, P.: Nonlinear Ship Motions, Proc. 3rd Int. Conf. on Num. Ship hydrodyn., Paris (1981), pp. 257-268.

8) Cointe, R., Geyer, P., King, B., Molin, B. and Tramoni, M. : Nonlinear and Linear Motions of a Rectangular Barge in a Perfect Fluid, Proc. 18th Symp. on Nav. Hydrodyn., Ann Arbor (1990), pp. 85-99.

9) Tanizawa, K. and Naito, S.: A Study on Parametric Roll Motions by Fully Nonlinear Numerical Wave Tank, Proc. 11th ISOPE Conf., Honolulu, Vol. 3 (1997), pp. 69-75.

10) Tanizawa, K. and Naito, S. : An Application of Fully Nonlinear Numerical Wave Tank to the Study on Chaotic Roll Motions, Proc. 12th ISOPE Conf., Montreal, Vol. 3 (1998), pp. 280-287. 